\title{
EFECTOS DE LA CONSTRUCCION DE PEQUEÑAS PRESAS EN CAUCES ANASTOMOSADOS DEL PIRINEO CENTRAL
}

\author{
José M. ${ }^{a}$ García-Ruiz* \\ Juan Puigdefábregas-Tomás**
}

\begin{abstract}
RESUMEN
En los ríos anastomosados del flysch eoceno pirenaico se han estudiado las consecuencias de la construcción de presas para reducir su torrencialidad $y$ arrastre de sedimentos. Aguas abajo de las presas aumenta la capacidad incisiva y de transporte; aguas arriba tiene lugar una ordenación espacial de los modelos sedimentarios, aunque la pendiente y la presencia de barras longitudinales pueden alterar dichos modelos.
\end{abstract}

\section{SUMMARY}

In braided streams of pyrenean eocen flysch we have studied the consequences of the building of dams to reduce their torrentiality and sediment load. Downstream the capacity of erosion and transport increases; upstream a spatial arrangement of sedimentary patterns takes place, though the gradient and the presence of longitudinal bars can alter such patterns.

En la bibliografía geográfica son numerosos los estudios llevados a cabo sobre la forma y dinámica de cauces fluviales. Existe asimismo interés creciente sobre la influencia de actividades humanas en cambios del régimen hidrológico, de aportes sedimentarios o de modelo de cauce. Ello no hace sino evidenciar el interés que los ejes de drenaje presentan desde un punto de vista geográfico al constituir su funcionamiento todo un compendio de las

* Departamento de Geografía. Colegio Universitario de La Rioja. Logroño.

** Instituto Pirenaico de Ecología. Jaca (Huesca). 
características de la cuenca hidrográfica y por la facilidad que presentan para adaptarse a cambios en tales características: alteración de la cubierta forestal, innovaciones en los usos agrarios del suelo, urbanización o construcción de presas. Estas últimas tienen el mismo significado, desde el punto de vista de la dinámica morfológica del lecho, que un cambio de régimen climático (LEOPOLD, WOLMAN and MILLER, 1964), sólo que en un espacio de tiempo muy breve.

Desde una perspectiva hidrológica y morfológica, los especialistas distinguen esencialmente tres tipos de lechos: rectos, meandriformes y anastomosados, con notables diferencias en el perfil longitudinal, la carga sedimentaria, pulsaciones de caudal y forma. De todos ellos quizás los anastomosados (braided streams) son los menos intensamente estudiados, aunque han sido descritos en ambientes muy diversos (GREGORY and WALLING, 1976). En el Pirineo Central español son frecuentes dentro del extenso afloramiento de flysch eoceno, donde han sido citados como elementos importantes en la interpretación del paisaje (GARCIA-RUIZ y PUIGDEFABREGAS, 1982). Con frecuencia, para evitar la torrencialidad y transporte de gravas, en estos ríos se han construido presas de pequeña entidad pero capaces de alterar la forma del cauce y el modelo de transporte/sedimentación. El objetivo del presente trabajo es identificar las consecuencias morfológicas que tienen esas presas de retención tanto aguas arriba como aguas abajo de la obra.

\section{El área de estudio}

El flysch eoceno surpirenaico ocupa una serie de alineaciones montañosas entre 600 y $2.200 \mathrm{~m}$. de altitud, al Sur de las Sierras Interiores y al Norte de la Depresión Interior en el Pirineo Central. La línea de cumbres corresponde a una serie de divisorias alomadas separadas por vertientes con pendiente entre 30 y $60 \%$. Lo esencial de la evolución del relieve está vinculado a una acción fluvial de diversa magnitud y a movimientos en masa. El desarrollo altitudinal del flysch permite distinguir tres ambientes clásicos (GARCIA-RUIZ y PUIGDEFABREGAS, 1982):

a) Las vertientes supraforestales, con cabeceras de torrente muy activas, solifluxión en suelos profundos y vertientes afectadas por una densa red de rigolas (rills).

b) Las vertientes forestales, con importante infiltración, formación de pipes y reptación.

c) Las vertientes submediterráneas degradadas y de cultivos abandonados, afectadas por la acción del hombre, con incisión de barrancos y arrastre de materiales, desprendimientos, rigolas, etc. 
AREA DE ESTUDIO

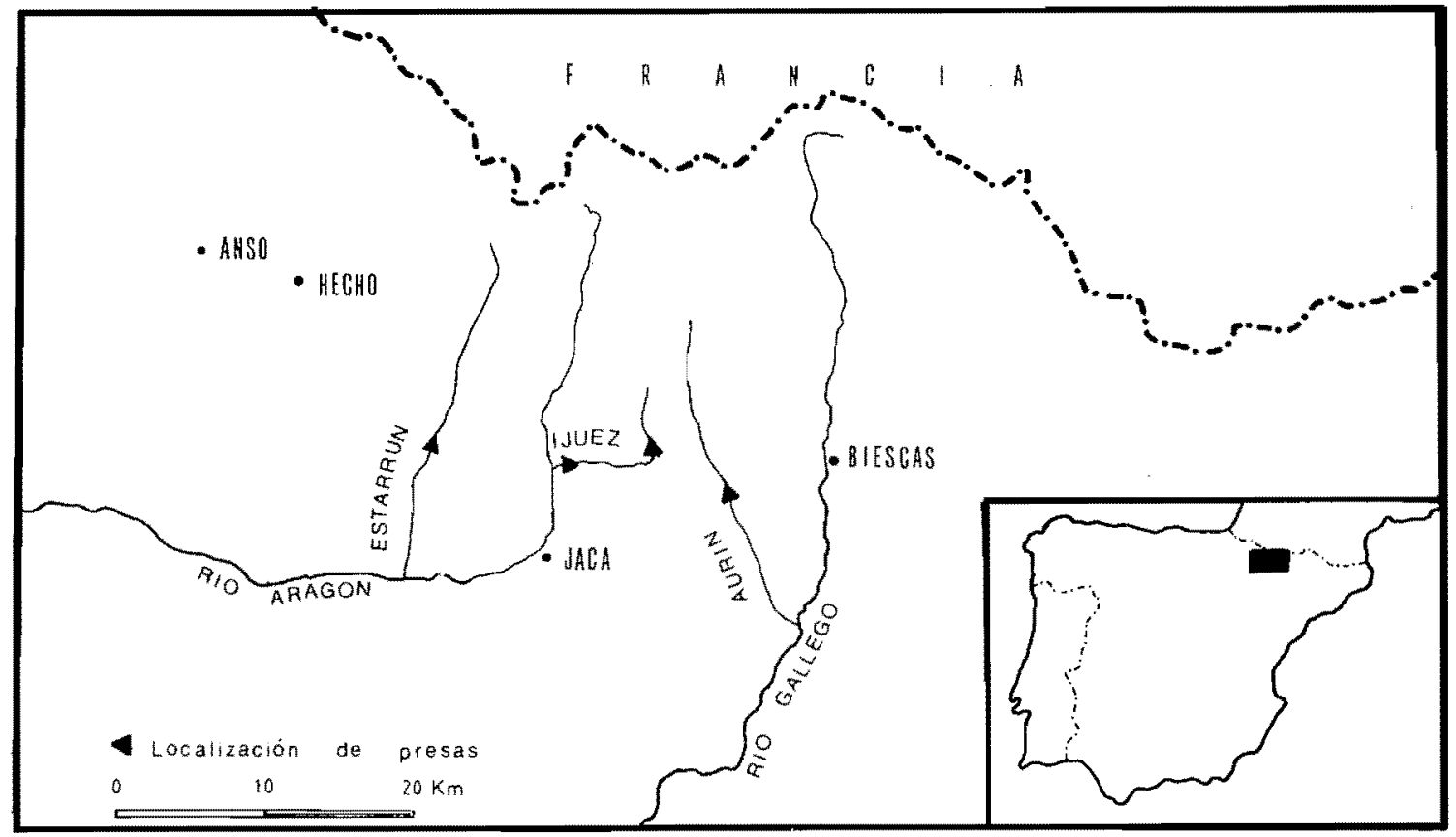

De estos tres ambientes, este último es el mejor condicionado para aportar grandes cantidades de sedimentos de tamaño muy heterogéneo hacia los cauces, en función de la importancia que presentan los desprendimientos, en forma de coladas de barro (mud flows) y de piedras (debris flows) (GARCIA-RUIZ y PUIGDEFABREGAS, 1984).

El clima puede definirse como de tendencia submediterránea con fuertes contrastes térmicos, una punta de sequía estival centrada en julio y dos máximos pluviométricos, uno en mayo y otro en noviembre. Las precipitaciones presentan valores absolutos similares a los de la altitud, de manera que, en la parte más alta, son de esperar unos $2.000 \mathrm{~mm}$. anuales. La intensidad de las lluvias no parece especialmente elevada (CREUS y PUIGDEFABREGAS, 1978 y ELIAS, 1963) y los aguaceros muestran preferencia por los meses de septiembre y noviembre.

\section{Métodos}

Se han seleccionado tres ríos con cauces anastomosados (ver gráf. 1): Estarrún, Ijuez y Aurin. Todos ellos cuentan con pequeñas presas de las que se han medido algunas características (Tabla 1). Aguas abajo de la presa se ha calculado sobre el terreno la sinuosidad, profundidad y anchura del lecho y la distancia a la que llega la influencia geomorfológica de la misma. Aguas arriba se han medido tamaños de cantos progresivamente más alejados de la presa, de forma continua y siempe a lo largo de un cauce para no mezclar 
la granulometría de diferentes ambientes sedimentarios. Una cinta colocada longitudinalmente en el cauce permitió seleccionar los cantos situados cada medio metro por debajo de aquélla, con lo que se dispuso de una perspectiva volumétrica acerca de la frecuencia con que aparecen los diferentes tamaños de cantos en el lecho.

\section{Los ríos anastomosados}

Aunque las condiciones climáticas en que aparecen pueden ser muy variadas, todos ellos presentan unos rasgos morfológicos y dinámicos similares. En opinión de RICHARDS (1982), son ríos dotados de elevada energía, con fuerte pendiente e importantes descargas de caudal; arrastran grandes cantidades de carga de fondo (bed load) muy heterométrica y las vertientes de gran parte de la cuenca muestran fuertes pendientes (LEOPOLD, WOLMAN and MILLER, 1964). Las orillas, por otra parte, carecen de cohesión, lo que favorece la ampliación del cauce y el abastecimiento fácil de materiales. Para TRICART y VOGT (1967) los factores decisivos son el tamaño y volumen de los sedimentos arrastrados y la irregularidad del caudal, sometido a pulsaciones instantáneas brutales.

Barras de diferentes formas y tamaños salpican el lecho; los canales cambian continuamente de posición, originando un trenzado (braid) de notable complejidad, que se transforma tras cada crecida; todo ello además alternando aportes de sedimentos nuevos con barras antiguas que unas veces son socavadas y otras semienterradas por barras más recientes.

Estas características se repiten en los ríos anastomosados del flysch pirenaico. Las laderas se hallan sometidas a muy frecuentes movimientos en masa, cuyas coladas llegan ocasionalmente hasta el cauce; por otra parte, el cultivo y la deforestación han provocado la instalación de barrancos con poderosa capacidad remontante, que desmantelan los antiguos campos cultivados o inciden entre el matorral, aportando grandes cantidades de materiales al lecho.

Con el fin de reducir la torrencialidad y frenar el aporte de materiales aguas abajo, se han construido presas de las llamadas de retención. Todas las estudiadas han perdido gran parte de su capacidad de almacenamiento, pues en su borde interno los sedimentos se hallan próximos al nivel del rebosadero de la presa. Sus características de forma y dimensiones responden a los modelos clásicos de presas de retención (GARCIA NAJERA, 1962), no sobrepasando en ningún caso los 7,50 m. de altura (vid Tabla 1). 
EFECTOS DE PRESAS EN CAUCES ANASTOMOSADOS

Tabla 1. Características de las presas e influencia de las mismas

\begin{tabular}{|l|c|c|c|c|c|c|c|c|c|c|}
\hline Presa & $\begin{array}{r}\text { Fecha de } \\
\text { construc. }\end{array}$ & $\begin{array}{c}\text { Altura } \\
\text { de presa }\end{array}$ & $\begin{array}{r}\text { Altura } \\
\text { rebosad. }\end{array}$ & $\begin{array}{c}\text { Nivel de } \\
\text { sediment. }\end{array}$ & (1) & (2) & (3) & (4) & (5) & (6) \\
\hline Estarrún & 1966 & 7,40 & 6,30 & 4,50 & 120 & 410 & 435 & 1,08 & 35,5 & 2,2 \\
Ijuez Bajo & 1958 & 6,65 & 6,00 & 4,65 & 165 & 515 & 460 & 1,09 & 15,0 & 2,0 \\
Ijuez Medio & 1966 & 6,50 & 5,75 & 5,75 & 20 & 195 & 175 & 1,02 & 13,0 & 1,2 \\
Aurín & 1966 & 6,00 & 4,80 & 4,80 & 77 & 600 & 480 & 1,16 & 15,0 & 1,0 \\
\hline
\end{tabular}

(1) Extensión ocupada por los sedimentos finos aguas arriba de la presa (en m.)

(2) Distancia aguas arriba de la influencia de la presa (en $\mathrm{m}$.)

(3) Distancia aguas abajo de la influencia de la presa (en m.)

(4) Sinuosidad del cauce en el tramo influido por la presa (aguas abajo)

(5) Anchura del cauce $60 \mathrm{~m}$. aguas abajo de la presa.

(6) Profundidad del cauce $60 \mathrm{~m}$. aguas abajo de la presa.

Tabla 2. Regresión múltiple paso a paso

Variable dependiente y : \% de gravas de menos de $10 \mathrm{~cm}$. de diámetro

Predictores $\mathrm{x}_{1}$ : distancia a la presa $(\mathrm{m})$

$\mathrm{x}_{2}$ : pendiente $(\%)$

\begin{tabular}{lll} 
Localidad & Predictores & Varianza explicada \\
\hline Ijuez Bajo & $1{ }^{\circ} \mathrm{x}_{1}$ & $0,403^{*}$ \\
& $2 .^{\circ} \mathrm{x}_{2}$ & $0,360^{* *}$ \\
& & $0,763^{*}$ \\
& $\mathrm{y}=-7,152 \mathrm{x}_{1}-0,161 \mathrm{x}_{2}+147,3$ \\
\hline Ijuez Medio & $1 .^{\circ} \mathrm{x}_{2}$ & $0,605^{*}$ \\
& $2 .^{\circ} \mathrm{x}_{1}$ & 0,100 \\
& & 0,705 \\
& $\mathrm{y}=-0,071 \mathrm{x}_{1}-3,842 \mathrm{x}_{2}=65,02$ \\
\hline
\end{tabular}

** Nivel de significación 0,01

* Nivel de significación 0,05 


\section{Los efectos de las presas aguas abajo}

El agua que sale de las presas ha dejado atrás prácticamente toda su carga sedimentaria. Se trata, pues, de un agua limpia, que ha cambiado sus características dinámicas al no tener que emplear parte de su potencia bruta en arrastrar sedimentos. El flujo resultante está dotado, por tanto, de gran energía, que puede emplear en adelante en incidir sobre su propio lecho o en socavar las orillas. PARK (1977) ha puesto de manifiesto esta capacidad de incisión, que puede llegar incluso a amenazar a obras de ingeniería próximas al cauce por socavamiento (STANLEY, 1972).

En las cuatro presas estudiadas se ha constatado la existencia de claros procesos de degradación. Se ha abierto un nuevo canal, más estrecho, encajado sobre la llanura aluvial construida antes de la presa y en muchos tramos se ha llegado hasta el sustrato rocoso; en las proximidades de la presa los cantos pequeños y medios (hasta $40 \mathrm{~cm}$.) han sido evacuados y sólo queda un armazón de grandes bloques. La anchura del nuevo cauce oscila entre 13 y $35,5 \mathrm{~m}$., delimitado por orillas abruptas, indicadoras de un proceso de encajamiento reciente. La profundidad del mismo oscila entre 1 y $2,2 \mathrm{~m}$. La antigüedad de la presa y la importancia de los caudales parecen influir en la mayor o menor importancia de la incisión. Con todo ello se confirma la "reducción de tamaño de los cauces comprobado por otros autores (PARK, 1977; GREGORY and PARK, 1974; GREGORY, 1976), al ajustarse la forma del canal a la nueva descarga dominante (ACKERS y CHARLTON, 1970), si bien conviene precisar que al pie de algunas presas se ha constatado la existencia de una canalización antrópica que, si no es determinante, al menos favorece momentáneamente el proceso de encajado.

Otro aspecto importante del problema es que, con la degradación, el modelo de lecho resultante es bien diferente del anterior. Se ha pasado de un cauce anastomosado a otro rectilíneo aunque con incipiente tendencia meandriforme. La sinuosidad es muy baja en todos los casos (v. Tabla 1), si bien en el Aurín se ha formado ya algún meandro bien incurvado. La pendiente del cauce, por su parte, disminuye levemente respecto a la situación anterior, aunque en algunos tramos se mantienen fuertes inclinaciones (de hasta el $7 \%$ ), mostrando el clásico esquema de riffles y pools.

Los efectos de la presa aguas abajo llegan hasta una distancia variable. En el Estarrún, Aurín e Ijuez Bajo la influencia llega hasta una distancia próxima a $500 \mathrm{~m}$. La desaparición de tal influencia se muestra por una apertura del cauce, que deja de estar confinado a un canal encajado, y la subdivisión del flujo en varios canales, retomando así el aspecto original. En la presa Media del Ijuez la influencia se pierde a los $180 \mathrm{~m}$., lo que es explicable pues la pendiente en este tramo fluvial es superior. 


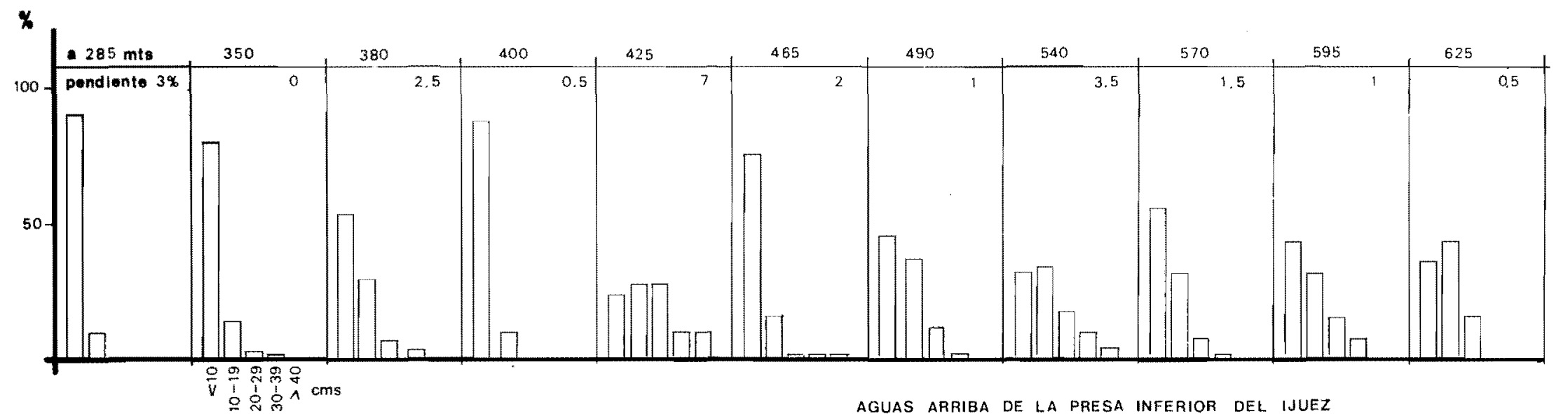

aguAS ARRIBA dE LA PRESA INFERIOR DEL IJUEZ 
JOSE M. ${ }^{a}$ GARCIA-RUIZ, JUAN PUIGDEFABREGAS-TOMAS
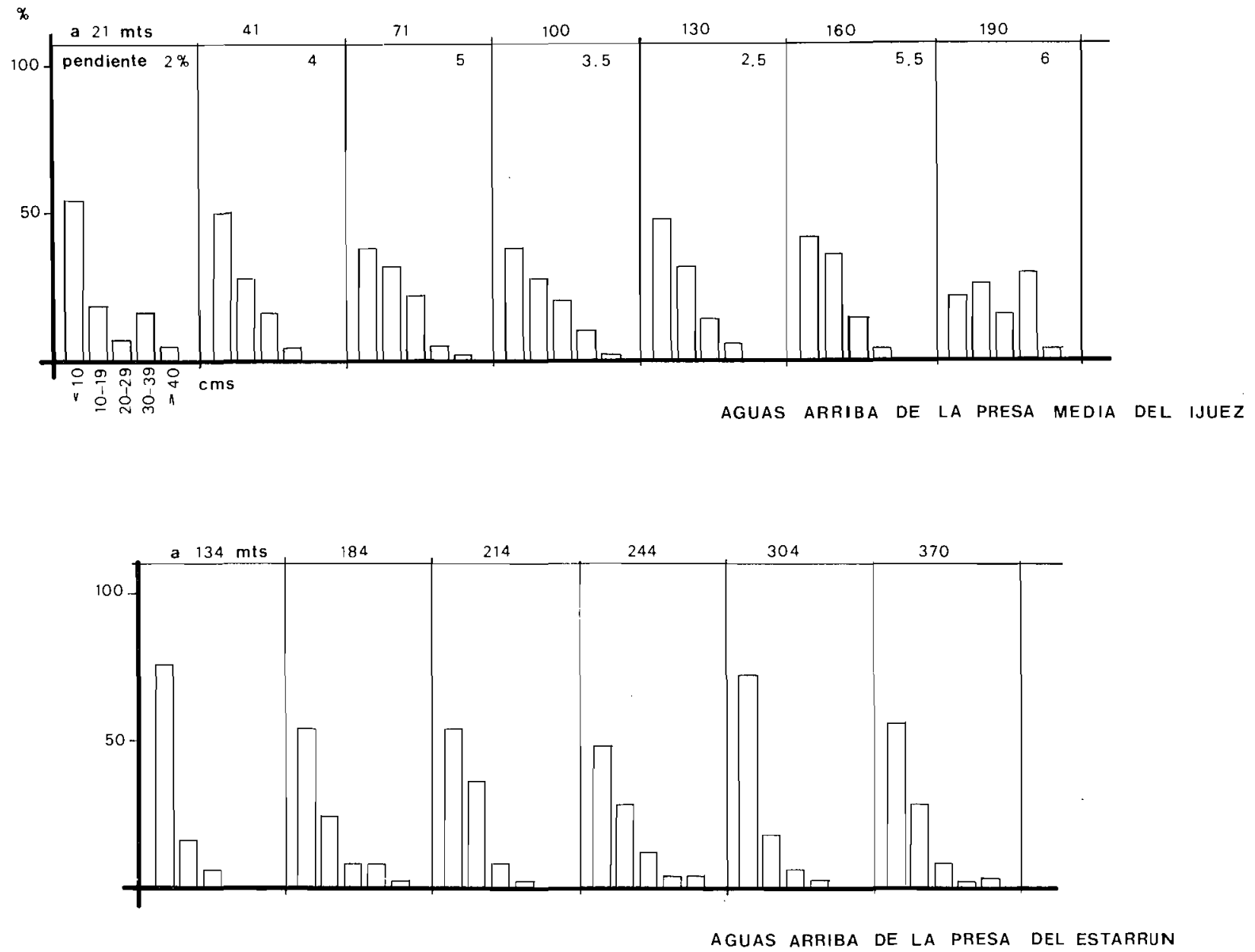


\section{Los efectos de las presas aguas arriba}

La instalación de una presa implica un cambio local del perfil del río con disminución de la pendiente y almacenamiento de agua. La consecuencia inmediata es el abandono de gran parte de la carga de sedimentos, al menos de la carga de granulometría más grosera. Nuestro objetivo es ver qué modelo se sigue en la distribución de tales sedimentos en el vaso de la presa y en qué medida influye la torrencialidad y carácter anastomosado de estos ríos en el referido modelo. La idea inicial de que partimos es que se tiene que producir una progresiva gradación en el tamaño de los sedimentos desde el borde interno de la presa - donde aparecen los más finos- hasta el límite aguas arriba de la influencia de dicha presa, donde la heterometría y el predominio de bloques ha de ser general, entrándose así aguas arriba en el dominio y características propios del cauce anastomosado.

Con este fin, a partir de las medidas de cantos, se han elaborado los gráficos 2 y 3 , donde mediante distribuciones de frecuencias puede apreciarse la evolución granulométrica hacia aguas arriba desde cada presa. Los histogramas comienzan a partir del momento en que aparecen gravas. La observación de los gráficos demuestra una marcada tendencia al predominio de gravas de menos de $10 \mathrm{~cm}$. en las proximidades de la presa; las gravas de ese tamaño disminuyen progresivamente aguas arriba, aunque la disminución no es lineal. Asimismo, aguas arriba existe una mayor tendencia a la heterometría, con mayor importancia de gravas de $10 \mathrm{a} 19 \mathrm{~cm}$. y aparición cada vez más frecuente de bloques de 30 a $39 \mathrm{~cm}$. -que se hacen dominantes en la presa Media del Ijuez - y de más de $40 \mathrm{~cm}$. Este último aspecto responde a lo esperado, pues frente a la complejidad del cauce normal, el efecto de la presa es simplificar la distribución de los sedimentos, eliminando primero los más gruesos, y después cada vez más pequeños conforme nos acercamos a la presa. En el modelo sencillo de la presa del Aurín, con datos a 1.000 y $77 \mathrm{~m}$., se ve el contraste claro entre un extremo y otro.

Sin embargo, el primero de los aspectos mencionados requiere una explicación. ¿Por qué en la progresión en el tamaño de los cantos - cualquiera que sea la clase - se producen saltos que rompen momentáneamente con la tendencia lineal? La misma observación de los gráficos permite comprobar que la pendiente parece desempeñar un papel importante: cuanto mayor es la pendiente, menor es la proporción de gravas pequeñas y mayor de bloques. Para evaluar la importancia relativa de la distancia a la presa y la pendiente sobre el control de la granulometría del cauce, hemos llevado a cabo un análisis de regresión múltiple paso a paso utilizando ambas variables como predictores y el tanto por ciento de gravas menores de $10 \mathrm{~cm}$. de diámetro como variable dependiente.

Los resultados (Tabla 2) muestran que, efectivamente, la distancia a la presa no es el único factor que influye en la clasificación sedimentaria de los 
JOSE M. ${ }^{a}$ GARCIA-RUIZ, JUAN PUIGDEFABREGAS-TOMAS

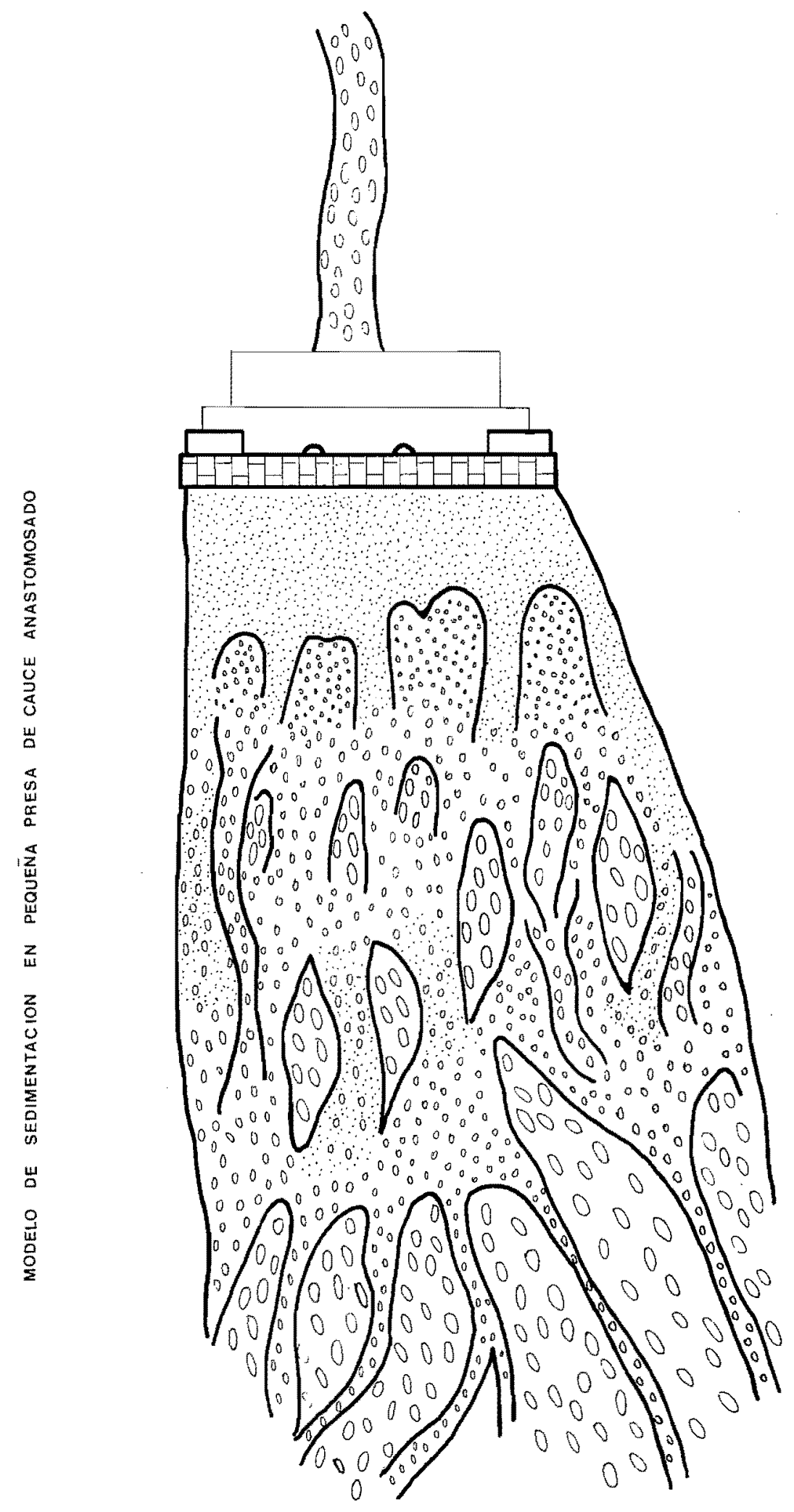




\section{EFECTOS DE PRESAS EN CAUCES ANASTOMOSADOS}

cantos. Más aún, el peso de la distancia es mayor en los tramos bajos, pero la situación parece invertirse hacia la cabecera, donde el papel de la pendiente local pasa a ser tan dominante que puede llegar a desdibujar la influencia de la primera.

La existencia de pequeñas rupturas de pendiente se debe $-y$ así lo hemos comprobado sobre el terreno- a la presencia de tramos en los que los cantos son más groseros que lo que teóricamente les correspondería. Los canales internos de la presa muestran, pues, un perfil típico de riffles y pools que refleja la inexistencia de una total linealidad en la progresión de tamaño de los cantos. Y esa alteración se debe a las propias características internas de la sedimentación en las presas.

En efecto, de forma esquemática presentamos un modelo de la distribución de sedimentos en el vaso de una presa. En el extremo de aguas arriba, el cauce posee el típico aspecto de un río anastomosado: varios canales sinuosos separados por barras longitudinales de materiales más gruesos. Al llegar al área de influencia de la presa los canales se disipan, en algunos casos con claro aspecto de desembocadura; el material se va haciendo más fino. Pero a la vez, entre los sedimentos propios de la presa aparecen pequeñas barras antiguas semienterradas que aportan localmente bloques gruesos a la sedimentación de la presa; es esa acumulación de bloques gruesos la que obliga localmente a aumentar la pendiente en los canales dispersos, alterándose así el modelo lineal de disminución del tamaño de cantos. Más cerca de ia presa, las gravas - ahora ya muy pequeñas - terminan en forma de lóbulos sobre los materiales finos que colmatan el borde interno de la presa.

En las presas de curso bajo - Ijuez Bajo y Estarrún- al espacio ocupado por los finos es muy importante; en las de curso alto los finos ocupan un espacio menor. Indudablemente la pendiente general del cauce juega aquí un papel importante. Allí donde la pendiente es fuerte -curso altola energía del flujo es suficientemente alta como para permitir el transporte de gravas hasta muy adentro de la presa. Allí la disminución de la pendiente es general y de ahí el cambio brusco de facies de gravas a finos.

Como consecuencia del relleno de la presa, la pendiente aguas arriba -que en el río anastomosado es muy fuerte, del orden del 3 y $4 \%$ en muchos tramos - se va suavizando lo que permite retener cada vez más sedimentos; ello implica que la tendencia a la simplificación sedimentaria se va prolongando hacia arriba, pues los materiales gruesos son retenidos cada vez más lejos de la presa. Dispersión de canales, enterramiento de barras antiguas, ausencia de nuevas barras bien formadas, organización de los sedimentos por tamaños y deposición caótica de cantos heterométricos al disminuir la pendiente en el extremo del área de influencia de la presa son, pues, las consecuencias más destacadas aguas arriba de la construcción de presas de retención. 


\section{Bibliografía}

ACKERS, P. and CHARLTON, F.G., 1970. The geometry of small meandering streams. Proc. Inst. Civ. Engrs., 73285, pp. 289-317.

CREUS, J. y PUIGDEFABREGAS, J., 1978. Influencia del relieve en la distribución de las precipitaciones máximas: un ejemplo pirenaico. Cuadernos de Investigación Geográfica, 4 (1): 11-23, Logroño.

ELIAS, F., 1963. Precipitaciones máximas en España. Ministerio de Agricultura. Servicio de Conservación de Suelos, 266 pp., Madrid.

GARCIA NAJERA, J.M., 1962. Principios de hidráulica torrencial. Instituto Forestal de Investigaciones y Experiencias, 297 pp., Madrid.

GARCIA-RUIZ, J.M. y PUIGDEFABREGAS, J., 1982. Formas de erosión en el flysch eoceno surpirenaico. Cuadernos de Investigación Geográfica, 8 (1-2): 85 128, Logroño.

GARCIA-RUIZ, J.M. y PUIGDEFABREGAS, J., 1984. Inestabilidad de laderas en el Pirineo Aragonés: Tipos de movimientos y su distribución geográfica. Jornadas sobre inestabilidad de laderas en el Pirineo. E.T.S.I. Caminos, 141-152, Barcelona.

GREGORY, K.J. and PARK, C.C., 1974. Adjustment of river channel capacity downstream from a reservoir. Water Resources Research, 10: 870-873.

GREGORY, K.J., 1976. Drainage basin adjustments and Man. Geographica Polonica, 34: 155-173.

GREGORY, K.J. and WALLING, D.E., 1976. Drainage basin. Form and process. E. Arnold, 458 pp., London.

LEOPOLD, L.B.; WOLMAN, M.G. and MILLER, J.P., 1964. Fluvial processes in Geomorphology. Freeman, 521 pp., San Francisco.

RICHARDS, K., 1982. Rivers. Form and processes in alluvial channels. Methuen and Co., 358 pp., London.

STANLEY, J.W., 1972. Retrogression on the lower Colorado River after 1935. Trans. Am. Soc. CN. Engrs., 116, Paper 2453, pp. 943-957.

TRICART, J. and VOGT, H., 1967. Quelques aspects du transport des alluvions grossières et du façonnement des lits fluviaux. Geografiska Annaler, 49A: 351366.

PARK, C.C., 1977. Man-induced changes in stream channel capacity. In River Channel Changes (Ed. by K.J. Gregory), Wiley, 121-144, Chichester. 